\title{
Present and future utility of computed tomography scanning in the assessment and management of COPD
}

\author{
Kristoffer Ostridge $\mathbb{1}^{1,2}$ and Tom M.A. Wilkinson ${ }^{1,2}$
}

\begin{abstract}
Affiliations:
${ }^{1}$ Southampton NIHR Respiratory Biomedical Research Unit, Southampton General Hospital, Southampton, UK. ${ }^{2}$ Clinical and Experimental Sciences, University of Southampton Faculty of Medicine, Southampton General Hospital, Southampton, UK.
\end{abstract}

\section{Correspondence:}

Tom Wilkinson, Clinical and Experimental Sciences, University of Southampton Faculty of Medicine, Sir Henry Wellcome Laboratories, Mailpoint 810, Southampton General Hospital, Tremona Road, Southampton, S016 6YD, UK.

E-mail: T.Wilkinsondsoton.ac.uk

ABSTRACT Computed tomography (CT) is the modality of choice for imaging the thorax and lung structure. In chronic obstructive pulmonary disease (COPD), it used to recognise the key morphological features of emphysema, bronchial wall thickening and gas trapping. Despite this, its place in the investigation and management of COPD is yet to be determined, and it is not routinely recommended. However, lung CT already has important clinical applications where it can be used to diagnose concomitant pathology and determine which patients with severe emphysema are appropriate for lung volume reduction procedures. Furthermore, novel quantitative analysis techniques permit objective measurements of pulmonary and extrapulmonary manifestations of the disease. These techniques can give important insights into COPD, and help explore the heterogeneity and underlying mechanisms of the condition. In time, it is hoped that these techniques can be used in clinical trials to help develop diseasespecific therapy and, ultimately, as a clinical tool in identifying patients who would benefit most from new and existing treatments. This review discusses the current clinical applications for CT imaging in COPD and quantification techniques, and its potential future role in stratifying disease for optimal outcome.

@ERSpublications

CT imaging has an important and rapidly developing role to play in the assessment of COPD http://ow.ly/ZREg3 


\section{Introduction}

Computed tomography (CT) has revolutionised medical imaging since it was developed in the 1970s. Its ability to provide detailed images of the lungs and thorax has made it an imaging modality of choice in respiratory medicine, where it has become an essential tool in the assessment and management of patients with many lung diseases.

In chronic obstructive pulmonary disease (COPD), CT can image the pathological changes that directly contribute to the airflow limitation that defines the condition [1]. Reliance on using spirometric markers to diagnose and characterise the disease has limited both our understanding of COPD and the individualised clinical management of the condition. COPD is a complex, heterogeneous condition with varying clinical features, consisting of a number of different phenotypes. The origins of clinical phenotypes and the associations of disease processes with endotypes along with the drivers to heterogeneity between individuals have not been fully explored, and are likely to have important implications for understanding disease progression and the future management of this condition [2]. There are currently no significant disease-modifying medications for COPD and there have not been the improvements in outcome seen in other chronic diseases. Other tools are required in addition to spirometry to help explain the heterogeneity, and provide further insights into approaches to study and treat COPD. CT has current practical applications in the routine management and investigation of the disease, and with the development of novel analysis techniques, has the potential to provide further details about the pulmonary and extrapulmonary manifestations of COPD.

\section{CT in current clinical practice}

Prior to the 1970s, histological and post mortem studies were required to study structural changes of the lung. The introduction of CT made it possible to visualise the thorax and assess lung structure noninvasively. In COPD, CT can identify key morphological features, including emphysema, bronchial wall thickening and gas trapping. These pathologies contribute directly to airflow obstruction and, therefore, CT has the potential to provide vital insights into the underlying pathophysiological changes of COPD. Despite this, routine CT imaging has not necessarily been widely adopted in clinical practice, and its place in the management and investigation of COPD has not been firmly established. The Global Initiative for Chronic Obstructive Lung Disease (GOLD) strategy does not routinely recommend CT scanning in COPD and only advises that it may be helpful in differential diagnosis or when surgical options are being considered [3]. This general ambivalence is highlighted by a study of attitudes in respiratory physicians and surgeons in the UK where only $32 \%$ thought it necessary for patients with severe COPD to have a CT scan [4].

There are a number of reasons why the use of CT has not become routine in COPD. One of the most important is the perceived notion that using CT does not alter management, although this is not strictly true. NETT (the National Emphysema Treatment Trial) demonstrated that patients with advanced upper lobe emphysema and limited exercise ability improved after lung volume reduction surgery [5]. There has also been considerable progress and experience in using endobronchial treatments for severe emphysema including coils, valves, sealant and airway bypass. In the correct patients with severe heterogeneous emphysema, these treatments can lead to significant improvement in pulmonary physiology and functional capacity $[6,7]$. CT imaging can also identify bullous disease that may be amenable to surgery and bullectomy can lead to improved health status [8]. It therefore seems clear that patients with severe COPD require CT imaging to determine which patients are appropriate for these techniques.

CT imaging can also detect concomitant pulmonary pathology in COPD. Bronchiectasis is particularly prevalent, with studies demonstrating 50\% of COPD patients having CT evidence of the disease [9-11]. When present, bronchiectasis is associated with worse airflow obstruction [11], increased exacerbations [9, 11], higher airway and systemic inflammation [9-11], and higher mortality [9]. COPD is also an independent risk factor for lung cancer [12], and higher cancer rates have been associated with worsening airflow obstruction [13, 14] and emphysema $[15,16]$. Fibrotic change has also been shown to be common in COPD and seems to confer a poor prognosis [17]. In addition, it can be difficult to diagnose due to the fact that pulmonary physiology may be normalised by the opposing effects of hyperinflation and fibrosis. As these concomitant pathologies are common and often associated with poorer outcomes, and would require a change management, it is vital that CT imaging is used to detect them.

A major limitation to the routine use of CT in COPD has been subjective reporting, making it difficult to interpret the results and guide management plans or give insights into the disease. Objective methods have been targeted to provide numerical data on key disease facets. Initially, simple visual scoring systems were developed, which showed early promise at quantifying the disease. These techniques were unable to detect subtle disease, and were liable to intra- and interobserver errors, as well as being insensitive to longitudinal change. To address these issues, automated quantitative analysis techniques have been developed, which 
are able to segment the lung parenchyma and airways from the chest wall and surrounding structures. Early software analysed two-dimensional axial slices but, coupled with advances in CT technology, a range of different analysis software exists that allows volumetric reconstructions (figure 1). This raises the prospect that quantitative CT analysis can be used to understand the disease and phenotypes in more detail, which the remainder of this review discusses.

\section{Emphysema}

Emphysema is traditionally a histological diagnosis, defined as an abnormal permanent enlargement of air spaces distal to the terminal bronchioles, accompanied by the destruction of alveolar walls. Emphysematous changes can be identified on CT and, in 1978, Rosenblum et al. [18] described areas of lower lung attenuation and mean lung density in patients with a clinical diagnosis of COPD.

To provide numerical data on emphysema, GoDDARD et al. [19] developed a visual scoring system for emphysema by estimating the amount on axial CT slices, which showed a strong association with airflow obstruction and with histological specimens [20-22]. Automated techniques were developed to segment the lung parenchyma and quantitate emphysema. The two main techniques both use the principle that emphysematous regions are represented by low-attenuation areas on CT. The first, termed CT densitometry, involves applying a density mask to the lung parenchyma and setting a threshold below which all voxels are assumed to be emphysema, giving a low-attenuation percentage (\%LAA) (figure 2). Initially, a threshold of -910 HU was used, which showed a strong correlation with pathological measures of emphysema [23]. Subsequently, \%LAA under $-950 \mathrm{HU}(\% \mathrm{LAA}<-950)$ was shown to have the strongest associations with both microscopic and macroscopic emphysema [24-27], leading to the common adoption of this threshold. The second method, termed percentile densitometry, involves choosing a threshold percentile in the attenuation distribution curve, which provides the density value in Hounsfield units under which a percentage of the voxels are distributed. A number of different thresholds between $1 \%$ and $18 \%$ have been used, and correlate strongly with microscopic emphysema on histological specimens [27]. The most commonly used threshold is $15 \%$ based on a number of studies in $\alpha_{1}$-antitrypsin deficiency $\left(\alpha_{1}\right.$-ATD) [28-30]. There is still no definite consensus about which of these methods is best and, even now, different studies use different thresholds. Given the fact that emphysema is an all-or-nothing phenomenon, it seems more intuitive to use CT densitometry, which uses an absolute cut-off to quantify it.

Studies have confirmed that CT densitometry demonstrates higher levels of emphysema in individuals with COPD than in healthy smokers [31-34]. There is also convincing evidence that CT densitometry has strong associations with airflow obstruction [10, 32-41], and analysis of over 4000 CT scans from the COPDgene study showed a strong negative correlation between \%LAA $<-950$ and forced expiratory volume in $1 \mathrm{~s}$ (FEV1), and demonstrated increased emphysema with worsening GOLD severity [33]. CT densitometry also has strong associations with gas transfer (transfer factor of the lung for carbon monoxide) [10, 35-40], 6-min walk distance (6MWD) [32, 36, 40, 42], BODE (body mass index, obstruction, dyspnoea, exercise capacity) score [36, 39, 43-45] and body composition [37, 39, 46, 47].

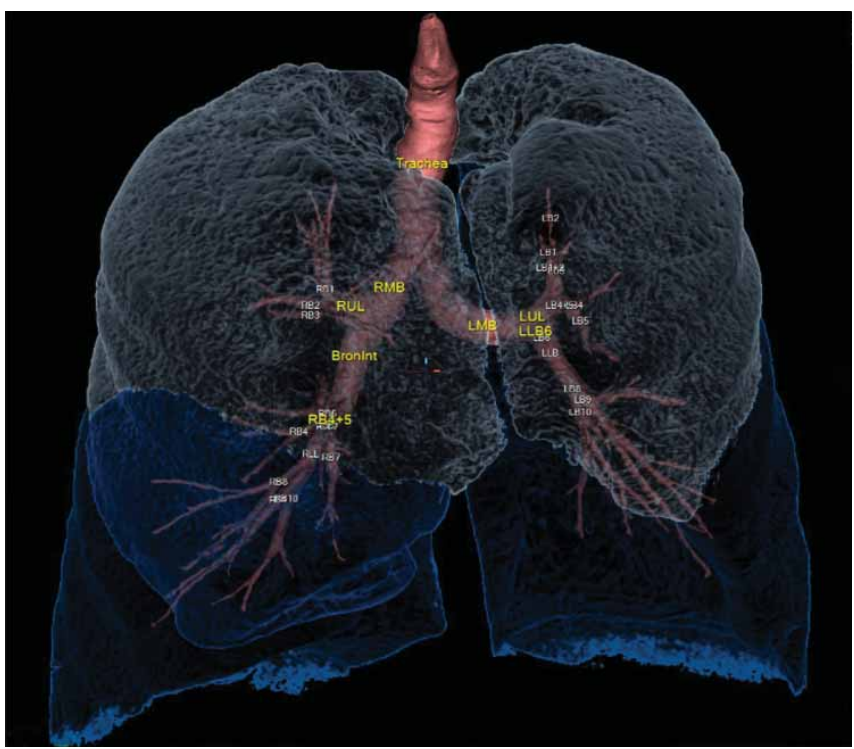

FIGURE 1 Volumetric computed tomography reconstruction of bronchial tree and lung parenchyma using Apollo software (VIDA Diagnostics Inc., Coralville, IA, USA). 
FIGURE 2 Coronal reconstruction of chest computed tomography showing low-attenuation areas below $-950 \mathrm{HU}$.

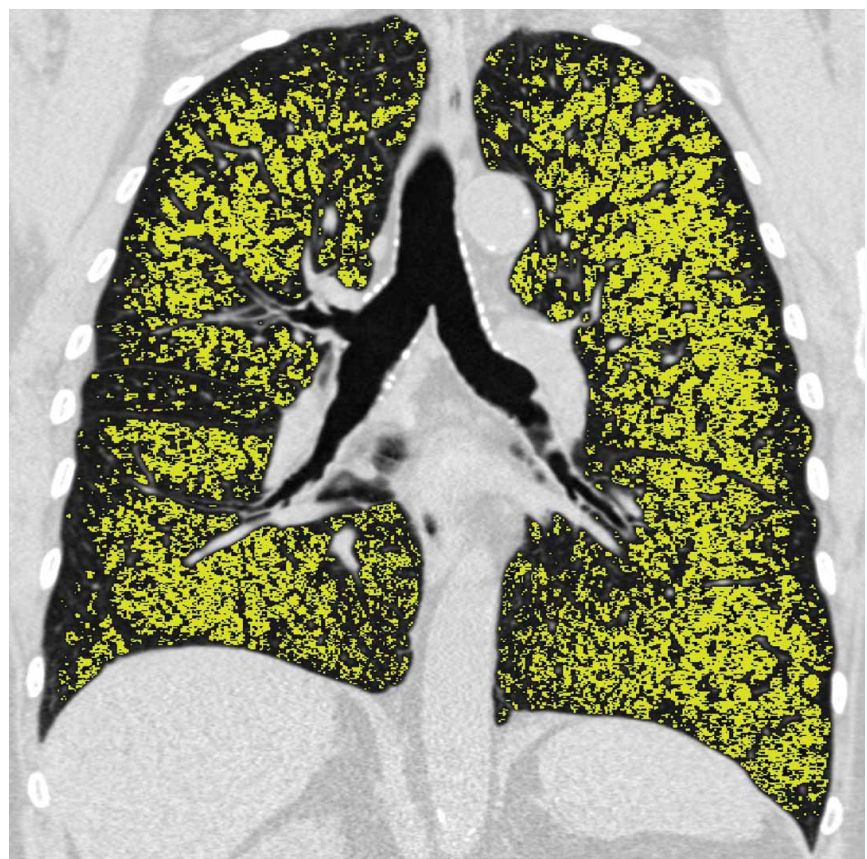

These densitometry techniques do not take into account the distribution or different subtypes of the disease, which may have important implications. Emphysema does not affect the lung in a uniform way and it is unclear how this affects the clinical presentation of the disease. As previously discussed, it has been shown to be clinically important in determining which patients benefit from lung volume reduction surgery [5]. A small study found that \%LAA was higher in central and upper regions within the lung [48]. Interestingly, airflow limitation correlated best with \%LAA in the central and lower regions, and also found that more homogenous emphysema was associated with worse airflow obstruction. Other studies have shown accelerated decline in lung function in those with homogenous emphysema [49] and those with more upper-zone emphysema [50]. With advances in quantitative CT analysis, lobar variation of disease can be assessed and significant differences in emphysema quantity between the lobes has been found $[51,52]$

Three distinct patterns of emphysema have been described in histological studies, based on the distribution within the secondary pulmonary lobule: centrilobular, panlobular and paraseptal emphysema. CT imaging can visualise these different subtypes. One study using a visual scoring system found that centrilobular emphysema was the most common subtype but over half of patients with emphysema had multiple subtypes [53]. An automated method, called local histogram-based emphysema patterns, involves dividing the lung into small cubes and analysing the subtype of emphysema present in each cube. This was found to be more predictive of lung function than \%LAA<-950 [54].

These data point to CT densitometry and other methods being able to accurately quantify emphysema and its association with physiological markers of disease. To validate CT imaging as a biomarker of disease, it is necessary to compare it to underlying disease mechanisms and outcome measures, and track longitudinal change. CT densitometry has been found to correlate with mortality in patients with COPD [55] and analysis from the GenKOLS trial [56] found that \%LAA had a better predictive value for respiratory and cardiac mortality than GOLD staging. Emphysematous change on CT has also been associated with higher exacerbation rate [57-59] and increased mortality from exacerbations [59].

A potentially important application of CT imaging is to track the changes in emphysema over time and see how it contributes to functional decline. Until recently, the majority of these studies were conducted in patients with $\alpha_{1}$-ATD and have shown that CT densitometry can accurately detect progression of emphysema [60-63] and correlates with decline in lung function [29,60]. Furthermore, CT lung density parameters are more sensitive in detecting progression of disease than traditional lung function [60] and have therefore been accepted as the primary end-point in prospective studies on treatment effects for $\alpha_{1}$-ATD. In COPD, it has been demonstrated that patients with higher quantities of emphysema on CT have an increased rate of decline of $\mathrm{FEV}_{1}[34,50]$. CT emphysema progression is also associated with frequent exacerbations [64] and current smoking status [65]. The largest longitudinal study to date assessed CT scans from the Evaluation of COPD Longitudinally to Identify Predictive Surrogate Endpoints 
(ECLIPSE) cohort [66], in which it was found that lung density and low-attenuation areas progressed on an annual basis. There were, however, significant variations between subjects and this did not convincingly correlate with lung function.

When comparing CT measures of emphysema with underlying markers of disease, it has to be considered that our knowledge of these mechanisms is imprecise. Emphysematous change has been associated with various matrix metalloproteinases (MMPs) [31, 67-69] as well as raised sputum neutrophils [70] and eosinophils $[39,71]$. A number of airway inflammatory cytokines, such as interleukin-8, leukotriene- $\mathrm{B}_{4}$ and myeloperoxidase, has been associated with emphysema progression [39, 72, 73] and emphysema has also been associated with raised serum fibrinogen [74]. Some work has been performed associating novel biomarkers with emphysema and in the ECLIPSE cohort, surfactant protein D and soluble RAGE (receptor of advanced glycation end-products) were both associated with decline in lung densitometry [66]. Little is known about bacterial colonisation and emphysema; however, BAFADHEL et al. [75] did not find any difference in bacteria culture between patients who had emphysema on CT and those who did not.

In summary, emphysema quantitation is relatively simple and can be performed with minimal user intervention. CT densitometry is associated with a number of physiological and clinical parameters. However, further work is required to evaluate emphysema distribution and subtypes, and to assess the longitudinal changes in emphysema, and the association with outcome markers of disease and underlying disease mechanisms.

\section{Bronchial wall measurements}

Airway wall remodelling is an important feature in COPD and histological specimens confirm airway wall thickening throughout the bronchial tree $[76,77]$. The limited resolution of CT means only large and intermediate-sized airways can be visualised directly. Early measurements of the airway relied on manual tracing [78]; however, a number of automated methods have been developed. One of these involves using the "full width at half maximum" principle (figure 3), which uses CT attenuation values to identify the inner and outer airway wall by projecting rays from the centre of the airway. These and other algorithms have been refined, and using region-growing approaches, it is now possible to generate three-dimensional reconstructions of the bronchial tree down to the fifth or sixth generation airway. Multiple airway dimensions can be measured from these reconstructions including bronchial wall thickness, wall area, lumen area, total airway area, wall area percentage (\%WA) and internal perimeter ( $P_{\mathrm{i}}$ ) (figure 4). A standardised parameter called $P_{\mathrm{i}_{10}}$ has been developed that predicts the square root of the wall area for a hypothetical airway with an internal perimeter of $10 \mathrm{~mm}$.

Early studies found that markers of bronchial wall thickening on CT were increased in COPD [46, 79-81]. However, more recent studies have shown that both airway wall and lumen size are reduced in COPD, although proportionally the lumen more so, resulting in a larger \%WA $[33,79,82]$. The reasons for the discrepancies are unclear although the later studies tended to make more complete measurements of the bronchial tree and spatially matched the airways. By not doing this, there is a chance of introducing bias into results by inadvertently selecting more proximal airways in COPD, which have thicker walls. This highlights the current difficulties in measuring the airways, with the sheer number of variables presenting significant challenges, and it is clear that further research is needed.

Taking the above limitations into account, measures of increased bronchial wall thickness are associated with FEV1 [41, 81, 83, 84] and this is stronger with more distal airways [41]. Bronchial wall markers also

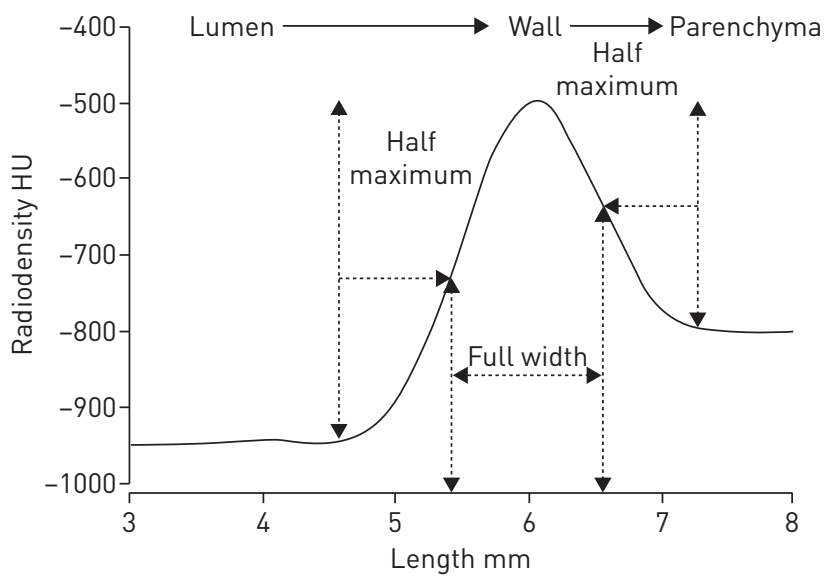

FIGURE 3 Full width at half maximum method for determining airway wall dimensions. 


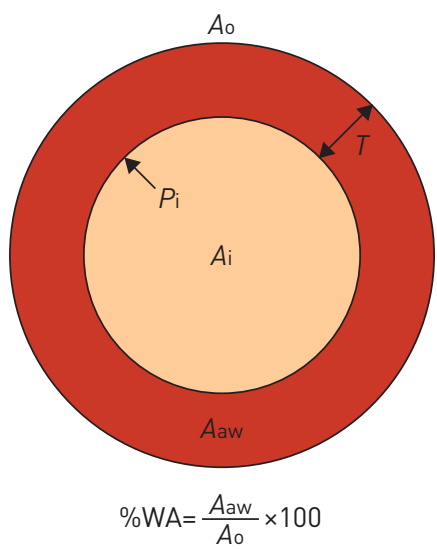

FIGURE 4 Airway wall dimensions. Aaw: airway wall area; T: wall thickness; Ao: total airway area; Ai: inner area; $P$ i: internal perimeter; \%WA: percentage wall area.

correlate with functional markers in the form of the BODE index [44, 45], exercise capacity [32, 36] and body composition [46]. Although statistically significant many of these associations tended to be quite weak. In the GenKOLS study, there was generally no increase in mortality with increased $P_{\mathrm{i}_{10}}$ but in a subgroup with severe emphysema, $P_{1_{10}}$ was associated with mortality [56]. Another study demonstrated that increased bronchial wall dimensions were associated with mortality from exacerbations [59]. It has been hypothesised that increased bronchial wall thickness is a marker of airway inflammation and therefore would be associated with symptoms of chronic bronchitis or frequent exacerbations. HAN et al. [57] confirmed this by showing that markers of airway wall thickness were increased in frequent exacerbators, although another study failed to find this association [58]. These measures also show associations with symptoms of chronic bronchitis $[84,85]$. Few studies have investigated the link between CT markers of bronchial wall thickening and sputum inflammatory markers or bacterial culture, although BAFADHEL et al. [75] did not find any association.

Simply measuring markers of bronchial wall thickness may be insufficient to describe the airway remodelling that occurs in COPD. Other methods have therefore been developed and one of these uses peak wall attenuation, which is a marker likely to reflect thickening and density within the airway wall. This has been shown to have a negative correlation with FEV1 $[41,86]$.

Minimal work has assessed the change of airway dimensions over time. A small study looked at lower lobe segmental airway changes between scans a year apart and did not find a significant difference, although did find a correlation between annual change in \%WA and FEV1 decline [87]. Regional variability in bronchial wall thickness has not been systematically analysed. OHARA et al. [88] did not find any difference between the apical upper lobe segmental bronchus and lower lobe segmental bronchus.

In theory, CT should be a useful tool at assessing the morphology of the large and intermediate-sized airways. However, there are still many uncertainties regarding this technology and measurements, and no definite evidence has shown that this is a particularly useful tool in COPD.

\section{Small-airway disease}

The small airways are the main contributor to the airflow obstruction seen in COPD [77], and histological studies confirm epithelial metaplasia, goblet cell hyperplasia and peri-bronchial fibrosis. There is currently no gold standard for measuring this small-airway disease and so better methods are required, and considerable research is ongoing in this area. CT cannot image the small airways directly as they are beyond the maximum resolution. The indirect sign of gas trapping can be used as a surrogate marker, which is defined as lack of volume reduction after expiration and is seen as low-attenuation areas on expiratory CT.

A number of quantification methods have been developed to measure gas trapping and the simplest of these involve calculating the number of voxels below $-856 \mathrm{HU}$ or the mean lung density (MLD) on expiratory CT. These methods do not compensate for the amount of emphysema and so, in effect, will be a combined score for gas trapping and emphysema. To correct for this, techniques using paired inspiratory and expiratory scans have been developed, which include using the ratio of MLD in expiration to inspiration. MAтSUоKa et al. [89] developed a more complex technique using the relative volume change between -856 and $-950 \mathrm{HU}$ between inspiratory and expiratory scans. This was validated in a study of 36 patients, and found strong associations with pulmonary function test markers of gas trapping and was superior to the other methods described above. When comparing these techniques to single-breath 
nitrogen washout, expiratory to inspiratory MLD ratio exhibited the best performance [90]. A large trial investigating gas trapping in COPDgene subjects found that paired (inspiratory and expiratory) samples were more predictive of pulmonary function in those with severe emphysema than just using the expiratory scan [91]. It therefore seems logical that any CT marker investigating gas trapping excludes emphysema by using paired inspiratory/expiratory scans.

Most studies investigating gas trapping have simply associated it to physiological parameters of disease where it has been found to correlate with FEV1 and other lung function parameters [31-33, 36, 38, 9294], and with worsening GOLD status [32, 92]. Few studies have investigated the link between gas trapping on CT and underlying mechanisms of disease. Our work has shown that CT markers of small-airway disease are associated with a number of MMPs (MMP-3, MMP-7, MMP-8, MMP-9, MMP-10 and MMP-12), suggesting these play a key role in small airways remodelling in COPD [31]. The longitudinal changes and the distribution of gas trapping throughout the lungs are unknown. More recently, novel CT methods of investigating small-airway disease have been developed, termed parametric response mapping (PRM), which involves co-registering inspiratory and expiratory scans so that voxel-by-voxel comparison can be performed (figure 5) [95]. This allows determination of emphysema and functional small-airway disease, and has been shown to subgroup patients effectively, which correlated with pulmonary function testing. Functional small-airway disease measured in the COPDgene cohort was independently associated with decline in FEV1 and this was especially the case with milder patients [96]. This and other similar techniques offer the opportunity to characterise disease phenotypes, and has the potential to monitor disease status, so considerable research is ongoing.

In summary, using paired inspiratory and expiratory scans, CT imaging can effectively measure gas trapping, although the optimum method has yet to be defined. Valid questions exist about whether gas trapping is truly a surrogate marker of small-airway disease, although it does show strong associations with pulmonary function measures of small-airway disease. Further work is needed to see how underlying mechanisms of disease associate with gas trapping and whether this parameter reflects changes in lung function over time.

\section{Other pulmonary morphological features}

There have been efforts to develop quantitative analysis for pulmonary features other than those discussed above. An automated method for bronchiectasis has been developed where the ratio of airway inner diameter to adjacent vessel diameter can be quantified [97]. This has shown the burden of bronchiectasis is higher with increasing airflow obstruction and is associated with increased exacerbations. Further work is required to refine and automate this technique.

There has also been interest in using CT-measured lung density as a marker of pulmonary inflammation. Increased lung density has been found in current smokers as opposed to never/ex-smokers [98-100], and has been associated with greater smoking history $[99,100]$ and inflammatory cells in bronchoalveolar lavage [98]. Interestingly, in a longitudinal study, lung density decreased after smoking cessation [100], suggesting smoking causes increased lung density, possibly through pulmonary inflammation. This may prove to be a potential tool in evaluating early lung disease prior to structural changes occurring in COPD.

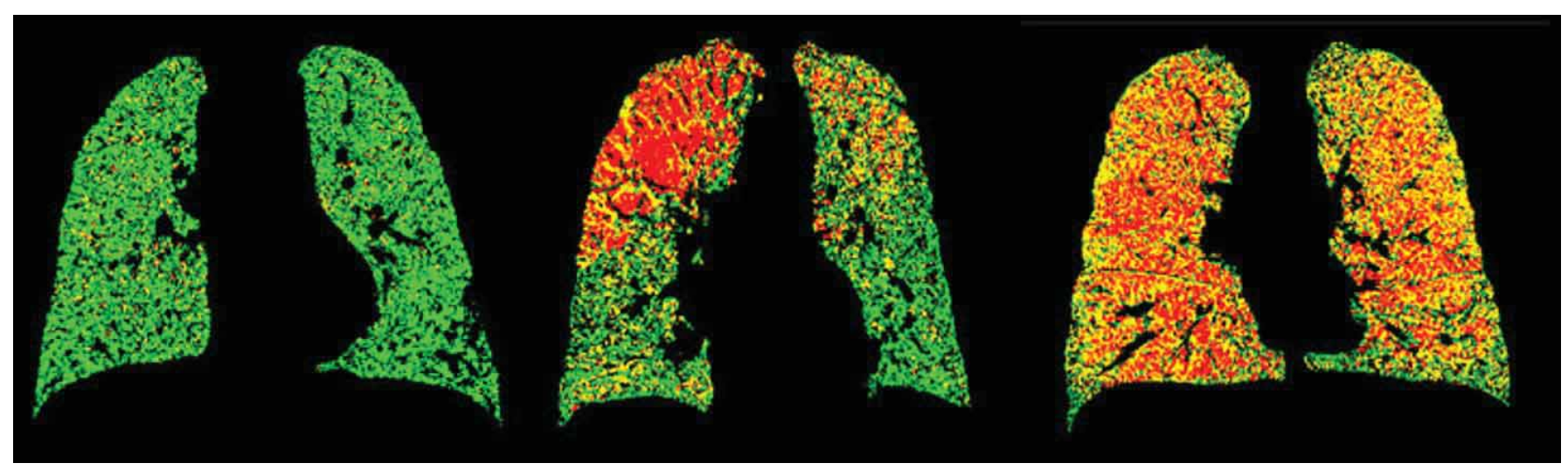

Normal tissue

Functional small-airway disease

Emphysema

FIGURE 5 Parametric response mapping from computed tomography scans in three individuals. Reproduced and modified from [95] with permission from the publisher. 
Pulmonary vascular imaging is an active area of research, as pulmonary vascular disease is well described in COPD and is a significant predictor of mortality [101-103]. The pulmonary artery diameter and ratio of pulmonary artery diameter to aorta diameter is associated with direct measures of pulmonary artery pressures [104] and higher exacerbation rates [105]. Cross-sectional area of small pulmonary vessels on CT is correlated strongly with invasive measures [106]. A technique to automatically segment and create a three-dimensional model of the pulmonary vasculature, allowing blood volume to be calculated, has been developed [107] and subjects with more emphysema had less blood volume. Further work is required to demonstrate the clinical utility of this.

\section{Extrapulmonary manifestations}

COPD has multiple systemic manifestations and CT of the chest is able to capture some of this information. Cachexia and skeletal muscle wasting are a significant problem in COPD, and body mass index (BMI) is associated with airflow obstruction, disease severity and mortality [108, 109]. There is growing recognition that other markers of body composition, such as fat-free mass index (FFMI), may be more important, as they are a better marker of skeletal muscle dysfunction. FFMI has been shown to be a predictor of mortality independently of fat mass or BMI $[110,111]$. A technique to assess pectoralis muscle area on CT showed significantly reduced muscle area in COPD versus healthy controls and was associated with GOLD staging [112]. This was also associated with functional markers of disease including the BODE index, MRC (Medical Research Council) dyspnoea score and 6MWD [112]. Various fat compartments can also be assessed including anterior chest wall subcutaneous fat and intra-abdominal fat, which have shown some clinical correlations. These methods are all relatively crude and still require significant user input.

Osteoporosis is also common in COPD [113] and bone mineral density has been identified as being independently associated with airflow obstruction $[114,115]$. Vertebral body attenuation values can be measured by CT and is associated with bone mineral density on dual-energy X-ray absorptiometry [116], and has also show associations with airflow obstruction [117] and exacerbation rate [118].

\section{Using CT to define phenotypes in COPD}

A main aim of COPD research is to define clinically meaningful phenotypes, and measuring morphological changes in isolation does not really help. More complex analysis that combines all of these morphological features is needed. One study separated patients into three subgroups: A phenotype had minimal emphysema, E phenotype had emphysema and no bronchial wall thickening, and $\mathrm{M}$ phenotype had both. A phenotype was associated with higher BMI, older age of onset of symptoms of breathlessness, fewer pack-years of smoking exposure and better lung function. Both $\mathrm{A}$ and $\mathrm{M}$ had more evidence of reversibility and higher sputum eosinophils. In another study, patients were split into emphysema-predominant and airway-predominant groups [57], and showed that emphysema patients had lower FEV1, and were more functionally limited, with higher BODE scores and lower BMI. CASTALDI et al. [119] performed cluster analysis on the COPDgene cohort incorporating quantitative CT analysis. They successfully grouped subjects into four clusters that had their own individual attributes. Other studies have also clustered subjects based on emphysematous change and bronchial wall thickening on CT scans [120]. Clinical applications of these phenotyping methods have thus far not been demonstrated.

\section{Other imaging modalities}

Although CT is the imaging modality of choice for providing structural images of the lung, one of the major drawbacks is its inability to provide functional imaging. CT can, however, be combined with other imaging modalities to provide functional data in COPD. Positron emission tomography (PET)-CT and single-photon computed tomography (SPECT) are nuclear medicine scans that combine radioisotopes with CT imaging, allowing both functional and molecular imaging studies with anatomical co-registration. Small molecules, peptides or proteins are labelled with radioactive atoms that emit high-energy photons, which can be detected by PET or SPECT. For PET-CT, ${ }^{18} \mathrm{~F}$-fluorodexoxyglucose (FDG) is the most commonly used radiolabel; it is taken up by metabolically active tissues and can be used as a marker of pulmonary neutrophilic inflammation. Uptake is significantly increased in COPD [121], and is associated with FEV1 and emphysema severity $[121,122]$. With SPECT, perfusion and ventilation imaging can be performed with either a vascular or inhaled tracer.

Magnetic resonance imaging (MRI) is another modality that can provide functional images of the lungs in COPD [123, 124]. There are a number of challenges in using magnetic resonance to image the lungs, including the low concentrations of protons, multiple air-tissue interfaces and relatively poor spatial resolution. Until recently, the main pulmonary application was in evaluating the pulmonary vasculature using contrast-enhanced imaging. Advances in technology, especially using oxygen-enhanced or hyperpolarised noble gases to increase the signal, allow functional imaging of ventilation and have shown ventilation defects in COPD [125]. Some studies have combined functional MRI with the structural images 
seen on CT. One such study assessed pulmonary microvascular blood flow, and found it was reduced in COPD and was associated with degree of emphysematous change [126]. Another study compared ventilation and perfusion defects measured on MRI with PRM measurements of emphysema and small-airway disease on CT. Ventilation defects were associated with emphysema and small-airway disease, and diffusion measurements were significantly raised in areas of gas trapping [127]. Magnetic resonance technology is still in early development but does provide an advantage over CT by allowing functional imaging and, importantly, being radiation free. Despite this, magnetic resonance is limited by availability, cost, the complexity of image analysis required and relatively poor spatial resolution.

\section{Future use of CT in research and management}

Further work is required to confirm quantitative CT analysis as an imaging biomarker of disease that can be used practically to manage patients with COPD and as a useful research tool to give insights into the disease. From a technical point of view, CT protocols need standardising as radiation dose, slice thickness and reconstruction algorithms can all effect analysis. Methods for quantifying the various morphological features need optimising, and this is especially the case for airway measurements and gas trapping. More sophisticated tools may be needed that do not just simply quantify results but also take into account other features, including emphysema subtypes and airway wall attenuation. Measures for detecting extrapulmonary manifestations are also in early development and further research is required to optimise these.

CT analysis needs to be assessed against outcome measures and markers of disease activity. To date, there have been relatively few longitudinal studies and these have shown variable results. It is vital studies are performed to track CT changes over time to get an accurate picture of the progression of the disease and how effective CT imaging is at capturing this. There have also been relatively few studies assessing quantifiable CT markers of disease and markers of underlying biological disease activity, which includes inflammatory markers, microbiology, proteases and other biomarkers. The approach taken in our study highlights how this can be achieved by combining bronchoscopic sampling and CT imaging to demonstrate a potentially important association between proteases and small-airway disease in COPD [31]. Similar approaches can be used to further our understanding of the active disease processes and endotypes occurring in different compartments and regions of the lung, hopefully allowing the development of novel targeted therapies.

Enhancing CT technology will also increase its application in COPD. A major limiting factor is the radiation dose and associated cancer risks. Dose reduction strategies are vital if longitudinal scans are to be considered. Dual-energy CT technology is being developed that allows functional imaging of the pulmonary vasculature and will have further applications. CT imaging could also be augmented with other imaging tools to provide more information about disease activity. PET-CT and SPECT are current examples, but these could be enhanced with development of novel molecular probes targeting key features of COPD. CT could also be co-registered with MRI to combine structural and functional imaging.

One of the most important questions is how to utilise CT analysis to define clinically important phenotypes. It is unlikely that simply subgrouping patients into emphysema and airway-predominant disease will be sufficiently helpful to guide treatment options. Cluster analysis and other statistical modelling techniques may provide interesting results but are more likely to be research tools with few practical clinical applications. Emphysema and bronchial wall thickening on CT have already been associated with increased mortality [55, 56, 59], and refining this information and combining it with clinical parameters may help enhance multidimensional prognostic tools such as the BODE index.

This technology may have a significant impact in clinical trial and drug discovery work in COPD. Quantitative CT has already been used as an outcome marker for emphysema progression in $\alpha_{1}$-ATD, and this could be extended for drugs targeting airway inflammation and small-airway disease. The heterogeneity of the disease causes significant challenges in clinical trial design. Most trials include unselected populations of patients. and therefore have to enrol large numbers and last considerable time to identify sufficient responders. Improving knowledge of patient heterogeneity is vital for optimising drug trials and quantitative CT may play an important role. Potentially, CT could be used to stratify study subjects into phenotypic groups that would be most likely to respond to the investigational drug. For example, roflumilast targets airway inflammation but study results have been underwhelming. CT could be used to define which patients have evidence of airway thickening/inflammation and this subject group could be used in any interventional study. Improving stratification of study subjects may increase treatment effects and reduce variability in response, thereby increasing the chances of novel drugs being developed. This will, in turn, lead to further clinical applications for CT imaging in determining which patients may benefit from novel pharmaceutical agents.

\section{Conclusion}

CT imaging has an important and rapidly developing role to play in the investigation of COPD, where it can aid diagnosis and provide information on significant concomitant disease, and can be used to plan 
interventional strategies for those with severe disease. It is also able to image the key pulmonary and extrapulmonary manifestations of the disease but the challenge has been how to utilise this information. Quantitative analysis shows promise in objectively measuring these disease features, and many have associations with traditional clinical and physiological markers of disease. These techniques are likely to be used initially as an important research tool with which to understand disease heterogeneity and aid our understanding of the key biological mechanisms in COPD. This understanding will aid optimal patient selection into clinical trials and translation of these approaches into clinical practice may help guide individualised management strategies and improve outcomes.

\section{References}

1 Rabe KF, Hurd S, Anzueto A, et al. Global strategy for the diagnosis, management, and prevention of chronic obstructive pulmonary disease: GOLD executive summary. Am J Respir Crit Care Med 2007; 176: 532-555.

2 Lopez-Campos JL, Bustamante V, Muñoz X, et al. Moving towards patient-centered medicine for COPD management: multidimensional approaches versus phenotype-based medicine - a critical view. COPD 2014; 11: 591-602.

3 Global Initiative for Chronic Obstructive Lung Disease. Global Strategy for the Diagnosis, Managment, and Prevention of Chronic Obstructive Pulmonary Disease. www.goldcopd.org/uploads/users/files/WatermarkedGlobal \%20Strategy\%202016(1).pdf

4 McNulty W, Jordan S, Hopkinson NS. Attitudes and access to lung volume reduction surgery for COPD: a survey by the British Thoracic Society. BMJ Open Respir Res 2014; 1: e000023.

5 Fishman A, Martinez F, Naunheim K. A randomized trial comparing lung-volume-reduction surgery with medical therapy for severe emphysema. N Engl J Med 2003; 348: 2059-2073.

6 Deslee G, Klooster K, Hetzel M, et al. Lung volume reduction coil treatment for patients with severe emphysema: a European multicentre trial. Thorax 2014; 69: 980-986.

7 Sciurba FC, Ernst A, Herth FJF, et al. A randomized study of endobronchial valves for advanced emphysema. N Engl J Med 2010; 363: 1233-1244.

8 Palla A. Elective surgery for giant bullous emphysema: a 5-year clinical and functional follow-up. Chest $J$ 2005; 128: 2043.

9 Martínez-García M-A, de la Rosa Carrillo D, Soler-Cataluña J-J, et al. Prognostic value of bronchiectasis in patients with moderate-to-severe chronic obstructive pulmonary disease. Am J Respir Crit Care Med 2013; 187: 823-831.

10 Patel IS, Vlahos I, Wilkinson TMA, et al. Bronchiectasis, exacerbation indices, and inflammation in chronic obstructive pulmonary disease. Am J Respir Crit Care Med 2004; 170: 400-407.

11 Martínez-García MÁ, Soler-Cataluña JJ, Donat Sanz Y, et al. Factors associated with bronchiectasis in patients with COPD. Chest 2011; 140: 1130-1137.

12 Kornum JB, Sværke C, Thomsen RW, et al. Chronic obstructive pulmonary disease and cancer risk: a Danish nationwide cohort study. Respir Med 2012; 106: 845-852.

13 Calabrò E, Randi G, La Vecchia C, et al. Lung function predicts lung cancer risk in smokers: a tool for targeting screening programmes. Eur Respir J 2010; 35: 146-151.

14 Kishi K, Gurney JW, Schroeder DR, et al. The correlation of emphysema or airway obstruction with the risk of lung cancer: a matched case-controlled study. Eur Respir J 2002; 19: 1093-1098.

15 Zulueta JJ, Wisnivesky JP, Henschke CI, et al. Emphysema scores predict death from COPD and lung cancer. Chest 2012; 141: 1216-1223.

16 de Torres JP, Bastarrika G, Wisnivesky JP, et al. Assessing the relationship between lung cancer risk and emphysema detected on low-dose CT of the chest. Chest 2007; 132: 1932-1938.

17 Jankowich M, Rounds S. Combined pulmonary fibrosis and emphysema syndrome: a review. Chest 2012; 141: $222-231$.

18 Rosenblum LJ, Mauceri RA, Wellenstein DE, et al. Computed tomography of the lung. Radiology 1978; 129: 521-524.

19 Goddard PR, Nicholson EM, Laszlo G, et al. Computed tomography in pulmonary emphysema. Clin Radiol 1982; 33: 379-387.

20 Bergin C, Müller N, Nichols DM, et al. The diagnosis of emphysema. A computed tomographic-pathologic correlation. Am Rev Respir Dis 1986; 133: 541-546.

21 Hruban RH, Meziane MA, Zerhouni EA, et al. High resolution computed tomography of inflation-fixed lungs. Pathologic-radiologic correlation of centrilobular emphysema. Am Rev Respir Dis 1987; 136: 935-940.

22 Kuwano K, Matsuba K, Ikeda T, et al. The diagnosis of mild emphysema. Correlation of computed tomography and pathology scores. Am Rev Respir Dis 1990; 141: 169-178.

23 Muller NL, Staples CA, Miller RR, et al. "Density mask": an objective method to quantitate emphysema using computed tomography. Chest 1988; 94: 782-787.

24 Gevenois PA, De Vuyst P, de Maertelaer V, et al. Comparison of computed density and microscopic morphometry in pulmonary emphysema. Am J Respir Crit Care Med 1996; 154: 187-192.

25 Gevenois PA, De Vuyst P, de Maertelaer V, et al. Comparison of computed density and macroscopic morphometry in pulmonary emphysema. Am J Respir Crit Care Med 1995; 152: 653-657.

26 Bankier AA, De Maertelaer V, Keyzer C, et al. Thoracic imaging pulmonary emphysema: subjective visual grading versus objective quantification with macroscopic morphometry and thin-section CT densitometry. Radiology 1999; 211: 851-858.

27 Madani A, Zanen J, de Maertelaer V, et al. Pulmonary emphysema: objective quantification at multi-detector row CT - comparison with macroscopic and microscopic morphometry. Radiology 2006; 238: 1036-1043.

28 Stolk J, Ng WH, Bakker ME, et al. Correlation between annual change in health status and computer tomography derived lung density in subjects with $\alpha 1$-antitrypsin deficiency. Thorax 2003; 58: 1027-1030.

29 Parr DG, Stoel BC, Stolk J, et al. Validation of computed tomographic lung densitometry for monitoring emphysema in $\alpha 1$-antitrypsin deficiency. Thorax 2006; 61: 485-490.

30 Newell JD, Hogg JC, Snider GL. Report of a workshop: quantitative computed tomography scanning in longitudinal studies of emphysema. Eur Respir J 2004; 23: 769-775. 
Ostridge K, Williams N, Kim V, et al. Relationship between pulmonary matrix metalloproteinases and quantitative CT markers of small airways disease and emphysema in COPD. Thorax 2016; 71: 126-132.

Rambod M, Porszasz J, Make BJ, et al. Six-minute walk distance predictors, including CT scan measures, in the COPDGene cohort. Chest 2012; 141: 867-875.

Schroeder JD, McKenzie AS, Zach JA, et al. Relationships between airflow obstruction and quantitative CT measurements of emphysema, air trapping, and airways in subjects with and without chronic obstructive pulmonary disease. AJR Am J Roentgenol 2013; 201: W460-W470.

Mohamed Hoesein FAA, de Hoop B, Zanen P, et al. CT-quantified emphysema in male heavy smokers: association with lung function decline. Thorax 2011; 66: 782-787. from CT estimation of disease extent. Eur Radiol 2007; 17: 911-918.

Lee YK, Oh Y-M, Lee J-H, et al. Quantitative assessment of emphysema, air trapping, and airway thickening on computed tomography. Lung 2008; 186: 157-165.

Ogawa E, Nakano Y, Ohara T, et al. Body mass index in male patients with COPD: correlation with low attenuation areas on CT. Thorax 2009; 64: 20-25.

O’Donnell RA, Peebles C, Ward JA, et al. Relationship between peripheral airway dysfunction, airway obstruction, and neutrophilic inflammation in COPD. Thorax 2004; 59: 837-842.

Boschetto P, Quintavalle S, Zeni E, et al. Association between markers of emphysema and more severe chronic obstructive pulmonary disease. Thorax 2006; 61: 1037-1042.

Diaz AA, Valim C, Yamashiro T, et al. Airway count and emphysema assessed by chest CT imaging predicts clinical outcome in smokers. Chest 2010; 138: 880-887.

Yamashiro T, Matsuoka S, Estépar RSJ, et al. Quantitative assessment of bronchial wall attenuation with thin-section CT: an indicator of airflow limitation in chronic obstructive pulmonary disease. $A J R$ Am $J$ Roentgenol 2010; 195: 363-369.

Diaz AA, Bartholmai B, San R, et al. Relationship of emphysema and airway disease assessed by CT to exercise capacity in COPD. Respir Med 2011; 104: 1145-1151.

Han MK, Bartholmai B, Liu LX, et al. Clinical significance of radiologic characterizations in COPD. COPD 2009; 6: 459-467.

Martinez $\mathrm{CH}$, Chen Y-H, Westgate PM, et al. Relationship between quantitative CT metrics and health status and BODE in chronic obstructive pulmonary disease. Thorax 2012; 67: 399-406.

Camiciottoli G, Bigazzi F, Bartolucci M, et al. BODE-index, modified BODE-index and ADO-score in chronic obstructive pulmonary disease: relationship with COPD phenotypes and CT lung density changes. COPD 2012; 9: 297-304.

Rutten EPA, Grydeland TB, Pillai SG, et al. Quantitative CT: associations between emphysema, airway wall thickness and body composition in COPD. Pulm Med 2011; 2011: 419328.

de Torres JP, Bastarrika G, Zagaceta J, et al. Emphysema presence, severity, and distribution has little impact on the clinical presentation of a cohort of patients with mild to moderate COPD. Chest 2011; 139: 36-42.

Haraguchi M, Shimura S, Hida W, et al Pulmonary function and regional distribution of emphysema as determined by high-resolution. Respiration 1998; 77: 125-129.

Tanabe N, Muro S, Tanaka S, et al. Emphysema distribution and annual changes in pulmonary function in male patients with chronic obstructive pulmonary disease. Respir Res 2012; 13: 31.

Mohamed Hoesein FAA, van Rikxoort E, van Ginneken B, et al. Computed tomography-quantified emphysema distribution is associated with lung function decline. Eur Respir J 2012; 40: 844-850.

Revel M-P, Faivre J-B, Remy-Jardin M, et al. Automated lobar quantification of emphysema in patients with severe COPD. Eur Radiol 2008; 18: 2723-2730.

Bhatt SP, Sieren JC, Newell JD, et al. Disproportionate contribution of right middle lobe to emphysema and gas trapping on computed tomography. PLoS One 2014; 9: e102807.

Smith BM, Austin JHM, Newell JD, et al. Pulmonary emphysema subtypes on computed tomography: the MESA COPD study. Am J Med 2014; 127: 94.

Castaldi PJ, San José Estépar R, Mendoza CS, et al. Distinct quantitative computed tomography emphysema patterns are associated with physiology and function in smokers. Am J Respir Crit Care Med 2013; 188: 1083-1090. Haruna A, Muro S, Nakano Y, et al. CT scan findings of emphysema predict mortality in COPD. Chest 2010; 138: 635-640.

Johannessen A, Skorge TD, Bottai M, et al. Mortality by level of emphysema and airway wall thickness. Am J Respir Crit Care Med 2013; 187: 602-608.

Han MK, Kazerooni EA, Lynch DA, et al. Chronic obstructive pulmonary disease exacerbations in the COPDGene study: associated radiologic phenotypes. Radiology 2011; 261: 274-282.

Motegi T, Ishii T. A comparison of three multidimensional indices of COPD severity as predictors of future exacerbations. Int J Chron Obstruct Pulmon Dis 2013; 8: 259-271.

Jairam PM, van der Graaf Y, Lammers J-WJ, et al. Incidental findings on chest CT imaging are associated with increased COPD exacerbations and mortality. Thorax 2015; 70: 725-731.

Dirksen A, Dijkman JH, Madsen F, et al. A randomized clinical trial of $\alpha_{1}$-antitrypsin augmentation therapy. Am J Respir Crit Care Med 1999; 160: 1468-1472.

Dowson LJ, Guest PJ, Stockley RA. Longitudinal changes in physiological, radiological, and health status measurements in $\alpha_{1}$-antitrypsin deficiency and factors associated with decline. Am J Respir Crit Care Med 2001; 164: 1805-1809.

Zagers H, Vrooman HA, Aarts NJM, et al. Assessment of the progression of emphysema by quantitative analysis of spirometrically gated computed tomography images. Investig Radiol 1996; 31: 761-767. by annual CT. Acta Radiol 1997; 38: 826-832.

Tanabe N, Muro S, Hirai T, et al. Impact of exacerbations on emphysema progression in chronic obstructive pulmonary disease. Am J Respir Crit Care Med 2011; 183: 1653-1659.

Tanabe N, Muro S, Sato S, et al. Longitudinal study of spatially heterogeneous emphysema progression in current smokers with chronic obstructive pulmonary disease. PLoS One 2012; 7: e44993. 

determined by CT scanning and biomarker expression: a prospective analysis from the ECLIPSE study. Lancet Respir Med 2013; 1: 129-136.

67 D'Armiento JM, Goldklang MP, Hardigan AA, et al. Increased matrix metalloproteinase (MMPs) levels do not predict disease severity or progression in emphysema. PLoS One 2013; 8: e56352.

68 Chaudhuri R, McSharry C, Brady J, et al. Sputum matrix metalloproteinase-12 in patients with chronic obstructive pulmonary disease and asthma: relationship to disease severity. J Allergy Clin Immunol 2012; 129: 655-663.

69 Chaudhuri R, McSharry C, Spears M, et al. Sputum matrix metalloproteinase-9 is associated with the degree of emphysema on computed tomography in COPD. Transl Respir Med 2013; 1: 11.

70 Boschetto P, Miniati M, Miotto D, et al. Predominant emphysema phenotype in chronic obstructive pulmonary disease patients. Eur Respir J 2003; 22: 568-569.

71 Kitaguchi Y, Fujimoto K, Kubo K, et al. Characteristics of COPD phenotypes classified according to the findings of HRCT. Respir Med 2006; 100: 1742-1752.

72 Miller M, Cho JY, Pham A, et al. Persistent airway inflammation and emphysema progression on CT scan in ex-smokers observed for 4 years. Chest 2011; 139: 1380-1387.

73 Parr DG, White AJ, Bayley DL, et al. Inflammation in sputum relates to progression of disease in subjects with COPD: a prospective descriptive study. Respir Res 2006; 7: 136.

74 Papaioannou AI, Mazioti A, Kiropoulos T, et al. Systemic and airway inflammation and the presence of emphysema in patients with COPD. Respir Med 2010; 104: 275-282.

75 Bafadhel M, Umar I, Gupta S, et al. The role of CT scanning in multidimensional phenotyping of COPD. Chest 2011; 140: 634-642.

76 Tiddens HAWM, Bogaard JM, de Jongste JC, et al. Physiological and morphological determinants of maximal expiratory flow in chronic obstructive lung disease. Eur Respir J 1996; 9: 1785-1794.

77 Hogg JC, Chu F, Utokaparch S, The nature of small-airway obstruction in chronic obstructive pulmonary disease. 2004; 350: 2645-2653.

78 Senéterre E, Paganin F, Bruel JM, et al. Measurement of the internal size of bronchi using high resolution computed tomography (HRCT). Eur Respir J 1994; 7: 596-600.

79 Smith BM, Hoffman EA, Rabinowitz D, et al. Comparison of spatially matched airways reveals thinner airway walls in COPD. The Multi-Ethnic Study of Atherosclerosis (MESA) COPD Study and the Subpopulations and Intermediate Outcomes in COPD Study (SPIROMICS). Thorax 2014; 69: 987-996.

80 Grydeland TB, Dirksen A, Coxson HO, et al. Quantitative computed tomography: emphysema and airway wall thickness by sex, age and smoking. Eur Respir J 2009; 34: 858-865.

81 Achenbach T, Weinheimer O, Biedermann A, et al. MDCT assessment of airway wall thickness in COPD patients using a new method: correlations with pulmonary function tests. Eur Radiol 2008; 18: 2731-2738.

82 Washko GR, Diaz AA, Kim V, et al. Computed tomographic measures of airway morphology in smokers and never-smoking normals. J Appl Physiol 2014; 116: 668-673.

83 Kim WJ, Silverman EK, Hoffman E, et al. CT metrics of airway disease and emphysema in severe COPD. Chest 2009; 136: 396-404.

84 Patel BD, Coxson HO, Pillai SG, et al. Airway wall thickening and emphysema show independent familial aggregation in chronic obstructive pulmonary disease. Am J Respir Crit Care Med 2008; 178: 500-505.

85 Kim V, Han MK, Vance GB, et al. The chronic bronchitic phenotype of COPD: an analysis of the COPDGene study. Chest 2011; 140: 626-633.

86 Washko GR, Dransfield MT, Estépar RSJ, et al. Airway wall attenuation: a biomarker of airway disease in subjects with COPD. J Appl Physiol 2009; 107: 185-191.

87 Ohara T, Hirai T, Sato S, et al. Longitudinal study of airway dimensions in chronic obstructive pulmonary disease using computed tomography. Respirology 2008; 13: 372-378.

88 Ohara T, Hirai T, Sato S, et al. Comparison of airway dimensions in different anatomic locations on chest CT in patients with COPD. Respirology 2006; 11: 579-584.

89 Matsuoka S, Kurihara Y, Yagihashi K, et al. Quantitative assessment of air trapping in chronic obstructive pulmonary disease using inspiratory and expiratory volumetric MDCT. AJR Am J Roentgenol 2008; 190: 762-769. Bommart S, Marin G, Bourdin A, et al. Relationship between CT air trapping criteria and lung function in small airway impairment quantification. BMC Pulm Med 2014; 14: 29.

91 Hersh CP, Washko GR, Estépar RSJ, et al. Paired inspiratory-expiratory chest CT scans to assess for small airways disease in COPD. Respir Res 2013; 14: 42

92 Matsuoka S, Kurihara Y, Yagihashi K, et al. Quantitative assessment of peripheral airway obstruction on paired expiratory/inspiratory thin-section computed tomography in chronic obstructive pulmonary disease with emphysema. J Comput Assist Tomogr 2007; 31: 384-389.

93 Mets OM, Murphy K, Zanen P, et al. The relationship between lung function impairment and quantitative computed tomography in chronic obstructive pulmonary disease. Eur Radiol 2012; 22: 120-128.

94 Mets OM, Zanen P, Lammers J-WJ, et al. Early identification of small airways disease on lung cancer screening CT: comparison of current air trapping measures. Lung 2012; 190: 629-633.

95 Galbán CJ, Han MK, Boes JL, et al. Computed tomography-based biomarker provides unique signature for diagnosis of COPD phenotypes and disease progression. Nat Med 2012; 18: 1711-1716.

96 Bhatt S, Soler X, Wang X, et al. Association between functional small airways disease and FEV1 decline in COPD. Am J Respir Crit Care Med 2016 [In press DOI: 10.1164/rccm.201511-2219OC].

97 Diaz AA, Ross J, Washko G, et al. Assessment of bronchiectasis in COPD using quantitative CT: prevalence and association with exacerbations. Am J Respir Crit Care Med 2014; 189: A6633.

98 Karimi R, Tornling G, Forsslund H, et al. Lung density on high resolution computer tomography (HRCT) reflects degree of inflammation in smokers. Respir Res 2014; 15: 23.

99 Lederer DJ, Enright PL, Kawut SM, et al. Cigarette smoking is associated with subclinical parenchymal lung disease: the Multi-Ethnic Study of Atherosclerosis (MESA)-lung study. Am J Respir Crit Care Med 2009; 180: 407-414.

100 Ashraf H, Lo P, Shaker SB, et al. Short-term effect of changes in smoking behaviour on emphysema quantification by CT. Thorax 2011; 66: 55-60. 
101 Scharf SM, Iqbal M, Keller C, et al. Hemodynamic characterization of patients with severe emphysema. Am J Respir Crit Care Med 2002; 166: 314-322.

102 Vizza CD, Lynch JP, Ochoa LL, et al. Right and left ventricular dysfunction in patients with severe pulmonary disease. Chest 1998; 113: 576-583.

103 Thabut G, Dauriat G, Stern JB, et al. Pulmonary hemodynamics in advanced COPD candidates for lung volume reduction surgery or lung transplantation. Chest 2005; 127: 1531-1536.

104 Pérez-Enguix D, Morales P, Tomás JM, et al. Computed tomographic screening of pulmonary arterial hypertension in candidates for lung transplantation. Transplant Proc 2007; 39: 2405-2408.

105 Wells JM, Washko GR, Han MK, et al. Pulmonary arterial enlargement and acute exacerbations of COPD. N Engl J Med 2012; 367: 913-921.

106 Matsuoka S, Washko GR, Yamashiro T, et al. Pulmonary hypertension and computed tomography measurement of small pulmonary vessels in severe emphysema. Am J Respir Crit Care Med 2010; 181: 218-225.

107 Estépar RSJ, Kinney GL, Black-Shinn JL, et al. Computed tomographic measures of pulmonary vascular morphology in smokers and their clinical implications. Am J Respir Crit Care Med 2013; 188: 231-239.

108 Lainscak M, von Haehling S, Doehner W, et al. Body mass index and prognosis in patients hospitalized with acute exacerbation of chronic obstructive pulmonary disease. J Cachexia Sarcopenia Muscle 2011; 2: 81-86.

109 Landbo C, Prescott E, Lange P, et al. Prognostic value of nutritional status in chronic obstructive pulmonary disease. Am J Respir Crit Care Med 1999; 160: 1856-1861.

110 Schols AMWJ, Broekhuizen R, Weling-Scheepers CA, et al. Body composition and mortality in chronic obstructive pulmonary disease. Am J Clin Nutr 2005; 82: 53-59.

111 Vestbo J. Body mass, fat free body mass and prognosis in COPD patients from a random population sample. Am J Respir Crit Care Med 2005; 173: 79-83.

112 McDonald M-LN, Diaz AA, Ross JC, et al. Quantitative computed tomography measures of pectoralis muscle area and disease severity in chronic obstructive pulmonary disease. A cross-sectional study. Ann Am Thorac Soc 2014; 11: 326-334.

113 Jørgensen NR, Schwarz P, Holme I, et al. The prevalence of osteoporosis in patients with chronic obstructive pulmonary disease: a cross sectional study. Respir Med 2007; 101: 177-185.

114 Kjensli A, Mowinckel P, Ryg MS, et al. Low bone mineral density is related to severity of chronic obstructive pulmonary disease. Bone 2007; 40: 493-497.

115 Vrieze A, de Greef MHG, Wijkstra PJ, et al. Low bone mineral density in COPD patients related to worse lung function, low weight and decreased fat-free mass. Osteoporos Int 2007; 18: 1197-1202.

116 Romme EAPM, Murchison JT, Phang KF, et al. Bone attenuation on routine chest CT correlates with bone mineral density on DXA in patients with COPD. J Bone Miner Res 2012; 27: 2338-2343.

117 Romme EAPM, Murchison JT, Edwards LD, et al. CT-measured bone attenuation in patients with chronic obstructive pulmonary disease: relation to clinical features and outcomes. J Bone Miner Res 2013; 28: 1369-1377.

118 Kiyokawa H, Muro S, Oguma T, et al. Impact of COPD exacerbations on osteoporosis assessed by chest CT scan. COPD 2012; 9: 235-242.

119 Castaldi PJ, Dy J, Ross J, et al. Cluster analysis in the COPDGene study identifies subtypes of smokers with distinct patterns of airway disease and emphysema. Thorax 2014; 69: 415-422.

120 Cho MH, Washko GR, Hoffmann TJ, et al. Cluster analysis in severe emphysema subjects using phenotype and genotype data: an exploratory investigation. Respir Res 2010; 11: 30.

121 Subramanian DR, Jenkins L, Edgar R, et al. Assessment of pulmonary neutrophilic inflammation in emphysema by quantitative positron emission tomography. Am J Respir Crit Care Med 2012; 186: 1125-1132.

122 Torigian DA, Dam V, Chen X, et al. In vivo quantification of pulmonary inflammation in relation to emphysema severity via partial volume corrected ${ }^{18}$ F-FDG-PET using computer-assisted analysis of diagnostic chest CT. Hell J Nucl Med 2013; 16: 12-18.

123 Sheikh K, Coxson HO, Parraga G. This is what COPD looks like. Respirology 2016; 21: 224-236.

124 Hoffman EA, Lynch DA, Barr RG, et al. Pulmonary CT and MRI phenotypes that help explain chronic pulmonary obstruction disease pathophysiology and outcomes. J Magn Reson Imaging 2016; 43: 544-557.

125 Parraga G, Ouriadov A, Evans A, et al. Hyperpolarized ${ }^{3} \mathrm{He}$ ventilation defects and apparent diffusion coefficients in chronic obstuctive pulmonary disease. Invest Radiol 2007; 42: 384-391.

126 Hueper K, Vogel-Claussen J, Parikh MA, et al. Pulmonary microvascular blood flow in mild chronic obstructive pulmonary disease and emphysema: the MESA COPD study. Am J Respir Crit Care Med 2015; 192: 570-580.

127 Capaldi D, Zha N, Guo F, et al. Pulmonary imaging biomarkers of gas trapping and emphysema in COPD: ${ }^{3} \mathrm{He}$ MR imaging and CT parametric response maps. Radiology 2016; 279: 579-608. 